d'animaux qui rachètent leur manque d'homogénéité par une parfaite adaptation au milieu.

Dans cette môme zonc côtière, dont le climat paraît bien convenir à la race hollandaise, existe un élevage de vaches à peu près pures. Le propriétaire utilise la race hollandaise depuis 1930. Les bêtes, élevées en demi-stabulation, sont fortement charpentées; jusqu'à ce jour elles ont été pratiquement indemnes de tuberculose et de piroplasmoses; bien nourries, leur rendement laitier est de 3.500 litres, en moyenne.

Quels enseignements pouvons-nous retirer de ces diverses observations?

Les méthodes de croisement, de neétissage à partir de la race hollandaise ne présentent pas grand intérêt. Eń effet, ou bien il s'agit d'une exploitation parfaitement bien équipée; dans ce cas, c'est l'animal de race pure, l'outil le plus perfectionné pour la transformation en lait des produits végétaux, qui est le plus susceptible d'assurer la rentabilité de la production laitière; ou bien ces conditions n'existent pas; dans ce cas, il est próférable d'utiliser des races douées de plus de rusticité.

Quant à l'importation de la race hollandaise au Maroc, elle peut être pratiquee avec succès, si certaines règles, qui seront énumérées plụs loin. sont appliquées.

Ce principe admis, quelle variété choisir?

Il viendrait assez facilement à l'esprit de fixer son choix sur la vache pie-rouge de Mouse-Rhin-Ysel, en raison de sa moins grande exigence au point de vue alimentaire et entretien. Cependant, comme l'importation de la race hollandaise ne peut être raisonnablement réalisée que dans le but d'une production laitière intensive, il semble indiqué de choisir résolument l'animal le plus perfectionné en vue de cette production, c'est-à-dire la pie-noire.

Pour prévenir un insuccòs total et cortain, l'importation de bétail hollandais est formellement déconseillee si les règles essentielles énumérées ci-après ne peuvent être strictement appliquées :

$1^{\circ}$ Date d'importation : Novembre et Décembre, pour permettre l'acclimatement, avant les fortes chaleurs.

$2^{\circ}$ Alimentation. : Rations d'entretien et de production suffisantes et équilibrée. Large place réservée au vert.

$3^{\circ}$ 'Hygiène. Etables en dur, crépies, faciles à désinfecter et désinsectiser. Ilygiène corporelle, douches froides en été.

$4^{\circ}$ Lutte contre les piroplasmoses.

$5^{\circ}$ Age des animaux. Vaches ou génisses pleines. Une importation de veaux s'est traduite par un échec complet.

\section{Conclusions.}

10 Actuollemiont le nombre d'exploitations susceptibles d'utiliser avec succès la race hollandaise est fort limité.

$2^{\circ} \mathrm{Ce}$ nombre peut etre notablement accru par l'amélioration possible des méthodes d'élevage dans certaines exploitations.

$3^{\circ}$ La large dispersion de la race hollandaise daris le monde n'étant pas le fait du hasard, mais étant due à sa faculté d'acclimatement et à ses hautes aptitudes laitières, il faut retenir que cette race bovine aura probablement un rôle à jouer, dans ce pays déficitaire en produits laitiers, et où demain, d'immenses périmètres irrigables devront être mis en valeur.

\title{
La race bovine noir-pie de Meknès
}

\author{
par M. GIRARD
}

LA race noir-pie de Meknès, si spéciale par sa robe, son caractère et son aire géographique, présente un réel intérêt zootechnique et commercial.

Le troupeau laitier indigène de Meknès et de ses environs immédiats compte dans la Médina et les villages voisins environ 2.000 animaux parmi lesquels on rencontre les robes suivantes : le noir, le noir-pie, le pie-noir à côté du fauve plus ou moins mêlé de noir. Les laitiers indigènes préfèrent et conservent jalousement les animaux de robe noir-pie, ils estiment surtoul les noirs à petites taches blanches.

Dès 1918-1922, des expériences du Service de
l'Elevage, lors de concours laitiers et beurriers, traduisaient par des chiffres le rendement de ces vaches; 6 litres d'un lait très riche en matières grasses après la mise bas pour des animaux d'un poids moyen de 225 kilos; 18 à 25 litres étaient nécessaires pour fabriquer un kilo de beurre.

Ces rendements sont remarquables si on tient compte du petit format des sujets et de l'absence de soins particuliers.

Vraisemblablement cette race, importée d'Europe au siècle dernier, a une filiation avec la Hollandaise, la Bretonne ou la Bordelaise et, depuis des générations, 
l'adaptation a un milieu sévère s'est effectuée avec tous ses bénéfices : rusticité, accoutumance au sol, à la végétation et au climat africains, absence de tuberculose, résistance exceptionnelle aux piroplasmes et aux maladies locales, si souvent fatales aux sujets importés de France.

Le Service de l'Elevage a entrepris de fixer les caractères zootechniques de la race créée par une circonstance fortuite et d'en améliorer les aptitudes laitières. En 1933, 22 veaux noir-pie étaient acquis non sans difficultés auprès des laitiers de la ville et la Station d'Essais de Meknès allait servir de cadre à cette expérimentation de longue haleine.

Les caractères généraux de la race noir-pie, que nous nous sommes attachés a fixer, sont les suivants :

Race de petit format, robe noir-pie, le blanc s'étendant largement sur la partie inférieure du corps, de l'interars jusqu'à la mamelle. L'écusson et la mamelle sont blancs parsemés de petites pigmentations circulaires noirâtres. La queue est blanche et noire ou complètement blanche. La croupe présente sur la ligne médiane une tache blanche en forme de losange large de 8 à 10 centimetres, longue de un à deux travers de main et pouvant s'étendre jusqu'à la base de la queue.

Cornes, onglons et muqueuses noirâtres.

Tête moyennement courte, à front plutôt haut, à profil droit avec orbites légèrement proéminentes, cornes' d'abord dirigées en avant et légèrement relevées à l'extrémité.

Mufle fin, encolure assez plate et fanon peu descendu, poitrine très développée, surtout chez le taureau, côte plutôt plate, dessus rectiligne, bassin large, ample, le plus possible horizontal, attache de la queue légèrement proéminente, membres bien d'aplomb, un peu enlevés, peau souple, fine, poils très noirs ou blancs purs.

Caractères laitiers prédominants. veines mammaires très développées, souvent six trayons.

Poids de l'animal adulte :

Taureau : 500-525 kilos.

Vache : $350-400 \mathrm{ktlos}$.

Tout en s'efforçant de conserver les caractères de rusticité indispensable dans une région aù climat pénible et aux ressources fourragères réduites, les pratiques mises en vigueur à la Station de Meknès ont surtout eu trait a l'hygiène de l'alimentation et de l'habitat. L'amélioration entreprise, parallelement par voie de sélection est une œuvire de longue haleine qui commence à porter ses fruits.

La production laitière s'est très sensiblement,accrue si on la compare aux chiffres cités plus haut, obtenus à partir d'animaux élevés et nourris chez les indigènes.

Dès 1939, soit six ans après le début de la sélection, les résultats obtenus étaient les suivants :

\begin{tabular}{|c|c|c|c|c|c|c|c|c|}
\hline MOIS & 1 & 2 & 3 & 4 & 5 & 6 & 7 & TOTAL EN 210 JOURS \\
\hline $\begin{array}{r}\text { Litres de lait vache } \\
\text { moyenne ......... } \\
\text { Litres de lait vache } \\
\text { d'élite ........... }\end{array}$ & 8,5 & 9,6 & 9,3 & 7,1 & 5,6 & 6 & 6 & $\begin{array}{c}1.366 \text { litres soit } 6 \text { I. } 5 \\
\text { de moyenne journalière } \\
1.600 \text { litres soit } 71.6 \\
\text { de moyenne journalière }\end{array}$ \\
\hline
\end{tabular}

A l'heure actuelle, à la l4 e année de l'expérience, voici quels sont les rendements :

\begin{tabular}{|c|c|c|c|c|c|c|c|c|}
\hline MOIS & 1 & 2 & & 4 & 5 & 6 & 7 & TOTAL EN 210 JOURS \\
\hline $\begin{array}{c}\text { Litres de lait vache } \\
\text { d'élite........... }\end{array}$ & 15 & 13 & 12 & 12 & 10 & 7 & 6 & $\begin{array}{l}2.280 \text { litres soit } 101.80 \\
\text { de moyenne journalière }\end{array}$ \\
\hline $\begin{array}{c}\text { Litres de lait vache } \\
\text { moyenne ......... }\end{array}$ & 10 & 9 & 9 & 8 & 7 & 6 & 5 & $\begin{array}{l}1.620 \text { litres soit } 7 \text { l. } 70 \\
\text { de moyenne journalière }\end{array}$ \\
\hline
\end{tabular}

On enregistre parallèlement à l'amélioration dans la production laitière une augmentation sensible dans l'ampleur et le format des animaux.

Ces résultats sont d'autant plus encourageants que le régime auquel est soumis le troupeau est le suivant : demi-stabulation, mise au pâturage toute l'année sur des prairies peu riches, ration complémentaire à l'étable assurée par le fourrage, l'orge en vert, la luzerne, le maïs fourrager et la betterave demi-sucrière, suivant la saison; exceptionnellement, les vaches en pleine lactation reçoivent une ration de son et de tourteaux. Dès l'âge de 15 jours, les veaux suivent leur mère au pâturage de manière à pouvoir têter à volonté et à satiété jusqu'au sevrage.

A signaler que, comme toutes les vaches indigènes, les mères donnent difficilement leur lait sans leur veau et sont pratiquement taries au $8^{\circ}$ mois de lactation.

De l'exposé ci-dessus on peut conclure que la race noir-pie peut, sans recevoir une rátion telle qu'elle est établie pour les races importées très améliorées, donner des rendements laitiers très intéressants. Cette race mérite d'être multipliée aux environs des villes, en particulier chez les laitiers ne disposant pas de grosses ressources fourragères. Elle est d'ailleurs très appréciée depuis que sa sélection a été entreprise à la Station d'Essais de Meknès; 136 géniteurs issus de notre troupeau ont été cédés aux éleveurs, tant européens que marocains. 